August 31, 1992

\title{
Free Field Representation of Quantum Affine Algebra $U_{q}\left(\widehat{\mathfrak{s l}}_{2}\right)$
}

\author{
Atsushi MATSUO \\ Department of Mathematics \\ Nagoya University, Nagoya 464-01, Japan
}

Abstract. A Fock representation of the quantum affine algebra $U_{q}\left(\widehat{\mathfrak{s l}}_{2}\right)$ is constructed by three bosonic fields for an arbitrary level with the help of the Drinfeld realization. 


\section{Introduction}

Recently the theory of the q-deformed chiral vertex operators (qVO) is developed by Frenkel and Reshetikhin [1] based on the representation theory of the quantum affine algebra $U_{q}(\hat{\mathfrak{g}})[2]$. They derive a q-difference equation for the n-point correlation function, which is a q-analoque of the Knizhnik-Zamolodchikov equation (KZ) in the Wess-ZuminoWitten (WZW) model [3] and is called the quantum Knizhnik-Zamolodchikov equation (qKZ). The importance of this model is due to the fact that some elliptic solutions of the quantum Yang-Baxter equation (YBE) of face type, including the ABF-solution [4], are obtained as the connection matrices of $\mathrm{qKZ}^{1}$.

For a detailed study of the model, a free field representation of $U_{q}(\hat{\mathfrak{g}})$ seems be an essential machinery. Jimbo et al. [7] explicitly calculate qVO for level one $U_{q}\left(\widehat{\mathfrak{s l}}_{2}\right)$-modules by making use of the free field representation obtained by Frenkel and Jing [8], which is a q-deformation of the Frenkel-Kac construction [10]. We note that the Drinfeld realization of $U_{q}(\hat{\mathfrak{g}})$ [11] is the main tool in their works. However a free field representation for an arbitrary level has not been known even in the $U_{q}\left(\widehat{\mathfrak{s l}}_{2}\right)$ case.

When $q=1$, the currents and the chiral vertex operators can be constructed by some free fields in general. In fact the Wakimoto representation [12] of the affine Lie algebra $\widehat{\mathfrak{s l}}_{2}$ is quite useful for the purpose. It is realized by one set of a $\beta-\gamma$ ghost system and a free bosonic field, and the vertex operators and the screening operators are explicitly written down [13]. This construction allows a generalization to the higher rank case [14], see also [16]. Remarkably, in the $\mathfrak{s l}_{2}$ case, the materials are also realized by three bosonic fields [15]. For instance, the standard $\mathfrak{s l}_{2}$ currents of level $k$ are expressed as

$$
\begin{gathered}
J^{ \pm}(z)=: \frac{1}{\sqrt{2}}\left[\sqrt{k+2} \partial \phi_{1}(z) \pm i \sqrt{k} \partial \phi_{2}(z)\right] e^{ \pm \sqrt{\frac{2}{k}}\left[i \phi_{2}(z)-\phi_{0}(z)\right]}:, \\
J^{0}(z)=-\sqrt{\frac{k}{2}} \partial \phi_{0}(z),
\end{gathered}
$$

where $\phi_{i}(z)$ are independent bosonic fields normalized as $\phi_{i}(z) \phi_{i}(w) \sim \log (z-w)$.

\footnotetext{
1 Aomoto et al. [5] have independently shown that the ABF-solution is obtained as the connection matrix of a q-difference equation, which is nothing else but a special case of qKZ [6].
} 
In this letter we shall generalize (1.1) to the case of the quantum affine algebra $U_{q}\left(\widehat{\mathfrak{s l}}_{2}\right)$. In section 2, we review the Drinfeld realization of $U_{q}\left(\widehat{\mathfrak{s l}}_{2}\right)$, whose generating functions play the role of the $\mathfrak{s l}_{2}$ currents. In section 3, we express them by means of three bosonic fields with slightly modified normalizations and expressions. Section 4 is devoted to a brief conclusion and discussion.

While completing this work the author learned that J. Shiraishi has also obtained a free field realization of $U_{q}\left(\widehat{\mathfrak{s l}}_{2}\right)$ for an arbitrary level in a different expression [19]. 


\section{Drinfeld realization of $U_{q}\left(\widehat{\mathfrak{s l}}_{2}\right)$}

Here and after we frequently use the notation

$$
[m]=\frac{q^{m}-q^{-m}}{q-q^{-1}} .
$$

The quantum affine algebra $U_{q}\left(\widehat{\mathfrak{s l}}_{2}\right)$ is isomorphic to the associative algebra generated by the letters $\left\{x_{m}^{ \pm} \mid m \in \mathbf{Z}\right\},\left\{a_{m} \mid m \in \mathbf{Z}_{\neq 0}\right\}, q^{ \pm \frac{c}{2}}$ and $q^{ \pm a_{0}}$, satisfying the following defining relations:

$$
\begin{gathered}
q^{ \pm \frac{c}{2}} \in \text { the center of the algebra, } \\
{\left[a_{m}, a_{n}\right]=\delta_{m+n, 0} \frac{[2 m][m c]}{m},\left[a_{m}, q^{a_{0}}\right]=0,} \\
q^{a_{0}} x_{m}^{ \pm} q^{-a_{0}}=q^{ \pm 2} x_{m}^{ \pm},\left[a_{m}, x_{n}^{ \pm}\right]= \pm[2 m] q^{\mp|m| c} x_{m+l}^{ \pm}, \\
x_{m+1}^{ \pm} x_{n}^{ \pm}-q^{ \pm 2} x_{n}^{ \pm} x_{m+1}^{ \pm}=q^{ \pm 2} x_{m}^{ \pm} x_{n+1}^{ \pm}-x_{n+1}^{ \pm} x_{m}^{ \pm}, \\
{\left[x_{m}^{+}, x_{n}^{-}\right]=\frac{1}{q-q^{-1}}\left(q^{\frac{(m-n)}{2} c} \psi_{m+n}-q^{\frac{(n-m)}{2} c} \varphi_{m+n}\right),}
\end{gathered}
$$

where $q^{ \pm m c}$ for $m>0$ is understood as $\left(q^{ \pm \frac{c}{2}}\right)^{2 m}$ and $\left\{\psi_{r}, \varphi_{s} \mid r \in \mathbf{Z}_{\geq 0}, s \in \mathbf{Z}_{\leq 0}\right\}$ are related to $\left\{a_{m} \mid m \in \mathbf{Z}_{\neq 0}\right\}$ by

$$
\begin{aligned}
& \sum_{m=0}^{\infty} \psi_{m} z^{-m}=q^{a_{0}} \exp \left(\left(q-q^{-1}\right) \sum_{m=1}^{\infty} a_{m} z^{-m}\right), \\
& \sum_{m=0}^{\infty} \varphi_{-m} z^{m}=q^{-a_{0}} \exp \left(-\left(q-q^{-1}\right) \sum_{m=1}^{\infty} a_{-m} z^{m}\right) .
\end{aligned}
$$

Now consider the following generating functions:

$$
\begin{gathered}
k_{+}(z)=\sum_{m=0}^{\infty} \psi_{m} z^{-m}, k_{-}(z)=\sum_{m=0}^{\infty} \varphi_{-m} z^{m} \\
x^{ \pm}(z)=\sum_{m \in \mathbf{Z}} x_{m}^{ \pm} z^{-m}
\end{gathered}
$$

Compositions of these operators are defined as a formal power series. Suppose that they act on a highest weight module and that $q^{\frac{c}{2}}$ acts by a scalar $q^{\frac{k}{2}}$ for some complex number 
$k$. Then we may understand them to be analytically continued outside some locus, and the operators (2.8) are characterized by the following properties:

$$
\begin{gathered}
{\left[k_{ \pm}(z), k_{ \pm}(z)\right]=0, \frac{q^{k+2} z-w}{q^{k} z-q^{2} w} k_{-}(z) k_{+}(w)=k_{+}(w) k_{-}(z) \frac{q^{-k+2} z-w}{q^{-k} z-q^{2} w},} \\
k_{+}(z) x^{ \pm}(w) k_{+}(z)^{-1}=\left(\frac{q^{\mp \frac{k}{2}}+2}{q^{\mp \frac{k}{2}} w-q^{2} z}\right)^{\mp 1} x^{ \pm}(w) \\
k_{-}(z) x^{ \pm}(w) k_{-}(z)^{-1}=\left(\frac{q^{\mp \frac{k}{2}+2} z-w}{q^{\mp \frac{k}{2}} z-q^{2} w}\right)^{ \pm 1} x^{ \pm}(w), \\
\left(z-q^{ \pm 2} w\right) x^{ \pm}(z) x^{ \pm}(w)=\left(q^{ \pm 2} z-w\right) x^{ \pm}(w) x^{ \pm}(z) \text { and } \\
x^{+}(z) x^{-}(w) \sim \frac{1}{q-q^{-1}}\left(\frac{z}{z-q^{k} w} k_{+}\left(q^{\frac{k}{2}} w\right)-\frac{z}{z-q^{-k} w} k_{-}\left(q^{-\frac{k}{2}} w\right)\right) .
\end{gathered}
$$

The last formula (opertor product expansion) means that $x^{+}(z) x^{-}(w)$ is analytically continued with the singular part being the right hand side and it coincides with $x^{-}(w) x^{+}(z)$. The defining relations (2.2)-(2.6) are recovered from these properties by a standard argument, see [9].

When $q$ goes to 1 , the algebra $U_{q}\left(\widehat{\mathfrak{s l}}_{2}\right)$ goes to the enveloping algebra $U\left(\widehat{\mathfrak{s l}}_{2}\right)$ of the affine Lie algebra $\widehat{\mathfrak{s l}}_{2}$, and the operators (2.8) go to the standard $\mathfrak{s l}_{2}$ currents by the following correspondence:

$$
x^{ \pm}(z) \rightarrow z J^{ \pm}(z), k^{+}(z)-k^{-}(z) \rightarrow 2 z J^{0}(z) .
$$




\section{Free field representation of $U_{q}\left(\widehat{\mathfrak{s l}}_{2}\right)$}

Let $k$ be a complex number. Let $\left\{\alpha_{\sigma}, \alpha_{\sigma}(n) \mid \sigma= \pm 1, n \in \mathbf{Z}\right\}$ be a set of operators satisfying the following relations:

$$
\left[\alpha_{\sigma}(m), \alpha_{\tau}(n)\right]=\sigma \delta_{\sigma, \tau} \delta_{m+n, 0} \frac{[2 m][k m]}{m}, \quad\left[\alpha_{\sigma}(m), \alpha_{\tau}\right]=\sigma \delta_{\sigma, \tau} \delta_{m, 0} .
$$

Here $\delta$ denotes the Kronecker symbol. Let $\left\{\beta(n) \mid n \in \mathbf{Z}_{\neq 0}\right\}$ be another set of operators satisfying

$$
[\beta(m), \beta(n)]=\delta_{m+n, 0} \frac{[2 m][(k+2) m]}{m}
$$

Suppose that they commute with $\alpha_{\sigma}$ and $\alpha_{\sigma}(m)$ for any $m$.

The Fock space $\tilde{\mathcal{F}}$ on which these operators act is supposed to be generated by the negative modes $\alpha_{1}(m), \alpha_{-1}(m), \beta(m)$, for $m<0$, and by $\alpha_{1}$ and $\alpha_{-1}$ acting on the vacuum vector $v$ satisfying the following conditions:

$$
\begin{gathered}
\alpha_{1}(m) v=\alpha_{-1}(m) v=\beta(m) v=0 \text { for any } m>0 \text {, and } \\
\alpha_{1}(0) v \text { and } \quad \alpha_{-1}(0) v \text { are scalar multiples of } v .
\end{gathered}
$$

We set

Wet

$$
\begin{gathered}
K_{+}(z)=\exp \left\{\left(q-q^{-1}\right) \sum_{m=1}^{\infty} z^{-m} \alpha_{1}(m)\right\} q^{\alpha_{1}(0)}, \\
K_{-}(z)=\exp \left\{-\left(q-q^{-1}\right) \sum_{m=1}^{\infty} z^{m} \alpha_{1}(-m)\right\} q^{-\alpha_{1}(0)}, \\
X^{+}(z)=\frac{1}{q-q^{-1}}\left\{Y^{+}(z) Z_{+}\left(q^{-\frac{k+2}{2}} z\right) W_{+}\left(q^{-\frac{k}{2}} z\right)\right. \\
\left.-W_{-}\left(q^{\frac{k}{2}} z\right) Z_{-}\left(q^{\frac{k+2}{2}} z\right) Y^{+}(z)\right\}, \\
X^{-}(z)=\frac{-1}{q-q^{-1}}\left\{Y^{-}(z) Z_{+}\left(q^{\frac{k+2}{2}} z\right) W_{+}\left(q^{\frac{k}{2}} z\right)^{-1}\right. \\
\left.-W_{-}\left(q^{-\frac{k}{2}} z\right)^{-1} Z_{-}\left(q^{-\frac{k+2}{2}} z\right) Y^{-}(z)\right\},
\end{gathered}
$$


where

$$
\begin{aligned}
& Y^{+}(z)= \exp \left\{\sum_{m=1}^{\infty} q^{-\frac{k m}{2}} \frac{z^{m}}{[k m]}\left(\alpha_{1}(-m)+\alpha_{-1}(-m)\right)\right\} \\
& e^{2\left(\alpha_{1}+\alpha_{-1}\right)} z^{\frac{1}{k}\left(\alpha_{1}(0)+\alpha_{-1}(0)\right)} \exp \left\{-\sum_{m=1}^{\infty} q^{-\frac{k m}{2}} \frac{z^{-m}}{[k m]}\left(\alpha_{1}(m)+\alpha_{-1}(m)\right)\right\}, \\
& Y^{-}(z)= \exp \left\{-\sum_{m=1}^{\infty} q^{\frac{k m}{2}} \frac{z^{m}}{[k m]}\left(\alpha_{1}(-m)+\alpha_{-1}(-m)\right)\right\} \\
& e^{-2\left(\alpha_{1}+\alpha_{-1}\right)} z^{-\frac{1}{k}\left(\alpha_{1}(0)+\alpha_{-1}(0)\right)} \exp \left\{\sum_{m=1}^{\infty} q^{\frac{k m}{2}} \frac{z^{-m}}{[k m]}\left(\alpha_{1}(m)+\alpha_{-1}(m)\right)\right\} \\
& Z_{+}(z)=\exp \left\{-\left(q-q^{-1}\right) \sum_{m=1}^{\infty} z^{-m} \frac{[m]}{[2 m]} \alpha_{-1}(m)\right\} q^{-\frac{1}{2} \alpha_{-1}(0)}, \\
& Z_{-}(z)=\exp \left\{\left(q-q^{-1}\right) \sum_{m=1}^{\infty} z^{m} \frac{[m]}{[2 m]} \alpha_{-1}(-m)\right\} q^{\frac{1}{2} \alpha_{-1}(0)}, \\
& W_{-}(z)=\exp \left\{\left(q-q^{-1}\right) \sum_{m=1}^{\infty} z^{m} \frac{[m]}{[2 m]} \beta(-m)\right\} \\
& W_{+}(z)=\exp \left\{-\left(q-q^{-1}\right) \sum_{m=1}^{\infty} z^{-m} \frac{[m]}{[2 m]} \beta(m)\right\}
\end{aligned}
$$

Proposition. By analytic continuation, $X^{ \pm}(z)$ and $K_{ \pm}(z)$ satisfy the same relations as $(2.9)-(2.12)$.

Proof. The relation (2.9) is obvious by the definition. The proofs of (2.10)-(2.11) are straightforward by calculating commutators of the fields. For example, the first relation of (2.10) follows from the following:

$$
\begin{aligned}
K_{+}(z) Y^{ \pm}(w) & =q^{ \pm 2} \exp \left\{ \pm \sum_{m=1}^{\infty} z^{-m} w^{m} q^{-\frac{k m}{2}} \frac{q^{2 m}-q^{-2 m}}{m}\right\} Y^{ \pm}(w) K_{+}(z) \\
& =\left(\frac{q^{2} z-q^{\mp \frac{k}{2}} w}{z-q^{2 \mp \frac{k}{2}} w}\right)^{ \pm 1} Y^{ \pm}(w) K_{+}(z) .
\end{aligned}
$$


Here we have used the formula: $\sum_{m=1}^{\infty} \frac{x^{m}}{m}=-\log (1-x)$. To prove (2.12) we put $X^{+}(z)=$ $\{A(z)-B(z)\} /\left(q-q^{-1}\right)$ and $X^{-}(z)=-\{C(z)-D(z)\} /\left(q-q^{-1}\right)$ where

$$
\begin{array}{ll}
A(z)=Y^{+}(z) Z_{+}\left(q^{-\frac{k+2}{2}} z\right) W_{+}\left(q^{-\frac{k}{2}} z\right), & B(z)=W_{-}\left(q^{\frac{k}{2}} z\right) Z_{-}\left(q^{\frac{k+2}{2}} z\right) Y^{+}(z), \\
C(z)=Y^{-}(z) Z_{+}\left(q^{\frac{k+2}{2}} z\right) W_{+}\left(q^{\frac{k}{2}} z\right)^{-1}, & D(z)=W_{-}\left(q^{-\frac{k}{2}} z\right)^{-1} Z_{-}\left(q^{-\frac{k+2}{2}} z\right) Y^{-}(z) .
\end{array}
$$

Then we have

$$
\begin{aligned}
A(z) C(w) & =\frac{q^{-1} z-q^{k+1} w}{z-q^{k} w} Y^{+}(z) Y^{-}(w) Z_{+}\left(q^{-\frac{k+2}{2}} z\right) Z_{+}\left(q^{\frac{k+2}{2}} w\right) W_{+}\left(q^{-\frac{k}{2}} z\right) W_{+}\left(q^{\frac{k}{2}} w\right)^{-1} \\
& \sim-\frac{\left(q-q^{-1}\right) z}{z-q^{k} w} Y^{+}\left(q^{k} w\right) Y^{-}(w) Z_{+}\left(q^{\frac{k}{2}-1} w\right) Z_{+}\left(q^{\frac{k}{2}+1} w\right) \\
& =-\frac{\left(q-q^{-1}\right) z}{z-q^{k} w} K_{+}\left(q^{\frac{k}{2}} w\right) .
\end{aligned}
$$

Similarly we have

$$
B(z) D(w) \sim \frac{\left(q-q^{-1}\right) z}{z-q^{-k} w} K_{-}\left(q^{-\frac{k}{2}} w\right),
$$

and the other products $A(z) D(w)$ and $B(z) C(w)$ are regular. The proof of the relation $\left[X^{+}(z), X^{-}(w)\right]=0$ is straightforward.

Q.E.D.

Now suppose that the vacuum vector $v$ of the Fock space $\tilde{\mathcal{F}}$ satisfies the condition

$$
\frac{1}{k}\left(\alpha_{1}(0)+\alpha_{-1}(0)\right) v=m v \text { for some integer } m
$$

Consider the subspace $\mathcal{F}$ of $\tilde{\mathcal{F}}$ generated by the actions of the negative modes and of $\alpha_{1}+\alpha_{-1}$ on $v$. Then it is clear by the definition that the mode expansion of $X^{ \pm}(z)$ and $K_{ \pm}(z)$ like (2.8) makes sense on $\mathcal{F}$ and that each Fourier component acts there. Thus we obtain a representation of the algebra $U_{q}\left(\widehat{\mathfrak{s l}}_{2}\right)$ on $\mathcal{F}$ with the level $c=k$. 


\section{Conclusion}

In this letter we have constructed the Fock representation of the quantum affine algebra $U_{q}\left(\widehat{\mathfrak{s l}}_{2}\right)$ for an arbitrary level $k$ in terms of three bosonic fields. In the $q \rightarrow 1$ limit

our representation goes to the representation of the affine Lie algebra $\widehat{\mathfrak{s l}}_{2}$ defined by the mode expansion of the currents (1.1). It is equivalent to a bosonization of the Wakimoto representation by a certain transformation [15].

In [16] solutions to $\mathrm{KZ}$ are explicitly constructed in the context of the Wakimoto representation, and they give rise to the integral solutions obtained previously [17]. The author expects that the Jackson integral solutions of qKZ [6,17] would be obtained in our formulation.

The present work will contribute to a better understanding of massive deformations of conformal field theory as the Wakimoto representation did in the WZW model. A detailed analysis of the representation and a construction of $\mathrm{qVO}$ and the screening operators will be contained in a separate paper.

\section{Acknowledgement.}

The author thanks K. Kimura, T. Miwa and J. Shiraishi for discussion. 


\section{References.}

[1] I.B. Frenkel and N.Yu. Reshetikhin, Commun. Math. Phys. 146 (1992) 1.

[2] V.G. Drinfeld, Sov. Math. Dokl. 32 (1985) 254.

M. Jimbo, Lett. Math. Phys. 10 (1985) 63.

[3] V.G. Knizhnik and A.B. Zamolodchikov, Nucl. Phys. B247 (1984) 83.

A. Tsuchiya and Y. Kanie, Adv. Stud. Pure. Math 16 (1988) 297.

[4] G.E. Andrews, R.J. Baxter and P.J. Forrester, J. Stat. Phys. 35 (1984) 193.

[5] K. Aomoto, Y. Kato and K. Mimachi, Int. Math. Res. Notes (Duke Math. J.) 65 (1992) 7 .

[6] A. Matsuo, Jackson integrals of Jordan-Pochhammer type and quantum KnizhnikZamolodchikov equations, preprint (1991) to appear in Commun. Math. Phys.

[7] M. Jimbo, K. Miki, T. Miwa and A. Nakayashiki, preprint RIMS-873 (1992).

[8] I.B. Frenkel and N.H. Jing, Proc. Nat'l Acad. Sci. USA 85 (1988) 9373.

[9] D. Bernard, Lett. Math. Phys. 17 (1989) 239.

[10] I.B. Frenkel and V.G. Kac, Invent. Math. 62 (1980) 23.

[11] V.G. Drinfeld. Sov. Math. Dokl. 36 (1987) 212.

[12] M. Wakimoto, Commun. Math. Phys. 104 (1986) 605.

[13] D. Bernard and G. Felder, Commun. Math. Phys. 127 (1990) 145.

[14] B. Feigin and E. Frenkel, Commun. Math. Phys. 128 (1990) 161.

P. Bouwknegt, J. McCarythy and K. Pilch, Prog. Theor. Phys. Suppl. 102 (1990) 67.

[15] D. Nemeschansky, Phys. Lett. B224 (1989) 121.

K. Ito. Nucl. Phys. B332 (1990) 566.

T. Jayaraman, K.S. Narain and M.H. Sarmadi, Nucl. Phys. B343 (1990) 418.

[16] G. Kuroki, Commun. Math. Phys. 142 (1991) 511.

H. Awata, A. Tsuchiya and Y. Yamada, Nucl. Phys. B365 (1991) 680.

[17] E. Date, M. Jimbo, A. Matsuo and T. Miwa, Intern. J. Mod. Phys. B4 (1990) 1049.

A. Matsuo, Commun. Math. Phys. 134 (1990) 65.

V.V. Schechtman and A.N. Varchenko, Invent. Math. 106 (1991) 139.

[18] A. Matsuo, Quatum algebra structure of certain Jackson integrals, preprint (1992). N.Yu. Reshetikhin, Jackson type integrals, Bethe vectors, and solutions to a difference analog of the Knizhnik-Zamolodchikov system, preprint (1992). 
[19] J. Shiraishi. Free boson representation of $U_{q}\left(\widehat{\mathfrak{s l}}_{2}\right)$, in preparation. 